Published Version: In: Proc IEEE Workshop on Applications of Signal Processing to Audio and Acoustics (WASPAA 2011), October 16-19, 2011, New Paltz, NY, USA, pp 261-264, 2011. doi:10.1109/ASPAA.2011.6082321

\title{
ON THE DISJOINTESS OF SOURCES IN MUSIC USING DIFFERENT TIME-FREQUENCY REPRESENTATIONS
}

\author{
Dimitrios Giannoulis, Daniele Barchiesi, Anssi Klapuri and Mark D. Plumbley \\ Centre for Digital Music, Queen Mary University of London, London, UK \\ E-mails: firstname.lastname@eecs.qmul.ac.uk
}

\begin{abstract}
This paper studies the disjointness of the time-frequency representations of simultaneously playing musical instruments. As a measure of disjointness, we use the approximate W-disjoint orthogonality as proposed by Yilmaz and Rickard [1], which (loosely speaking) measures the degree of overlap of different sources in the time-frequency domain. The motivation for this study is to find a maximally disjoint representation in order to facilitate the separation and recognition of musical instruments in mixture signals. The transforms investigated in this paper include the short-time Fourier transform (STFT), constant-Q transform, modified discrete cosine transform (MDCT), and pitch-synchronous lapped orthogonal transforms. Simulation results are reported for a database of polyphonic music where the multitrack data (instrument signals before mixing) were available. Absolute performance varies depending on the instrument source in question, but on the average MDCT with $93 \mathrm{~ms}$ frame size performed best.
\end{abstract}

Index Terms - Source separation, W-disjoint orthogonality, constant Q transform, MDCT, pitch-synchronous analysis

\section{INTRODUCTION}

The objective of blind source separation is to extract a set of source signals $\left\{\mathbf{s}_{k}\right\}_{k=1}^{K}$ from one or more observed mixtures $\left\{\mathbf{x}_{n}\right\}_{n=1}^{N}$ without prior information about the mixing process. When the number of observed mixtures $M$ is equal or greater than the number of sources $K$, the problem is said to be determined or overdetermined, respectively, and can be tackled with methods such as independent component analysis (strictly defined for the determined case).

On the other hand, when $K>N$ and usually $N=1$, the problem is underdetermined and can be solved only by making some assumptions on the sources. There are several approaches to underdetermined source separation and the use of time-frequency masks is a popular one among them. Estimating the masks from the mixture signal is a very hard task. There are different approaches for that, from auditory motivated (CASA)[?] to statistical parametric spectral models[2], and blind methods like non-negative matrix factorization (NMF) based sound separation approaches[3] which can be viewed as estimating soft-masks for sources.

The overall structure of a masking algorithm can be described as follows. A mixture $\mathrm{x}$ is first mapped by a linear operator $\mathcal{T}$ into a transformed domain, usually a time-frequency or time-scale representation. Then $K$ masks $\left\{M_{k}\right\}_{k=1}^{K}$ are estimated in order to extract the source signals. After each mask has been applied in the transformed domain, an estimate $\hat{\mathbf{s}}_{k}$ of the corresponding source $\mathbf{s}_{k}$ can be obtained by inverting the transform:

$$
\hat{\mathbf{s}}_{k}=\mathcal{T}^{-1}\left(M_{k}(\mathcal{T}(\mathbf{x}))\right)
$$

Choosing a suitable transform $\mathcal{T}$ is crucial for the success of the source separation algorithm. In particular, $\mathcal{T}$ must satisfy the following properties:

- Invertibility: the transform must admit an inverse in order to obtain the source signals in the time domain.

- Fast implementation: the transform must admit a fast implementation in order to be suitable for separation of audio sources from entire songs and even large databases of music.

- Disjoint representation: the transform must lead to a representation in which the support of the coefficients corresponding to different sources overlap as little as possible.

While the first two properties define a set of valid operators from which to choose from, the third property is the focus of the present work where we compare a range of transforms and measure the degree of disjointness of the different instruments in a music mixture. Previous work includes a paper by Tan and Fevotte [4] measuring the performance of a source separation algorithm using various orthonormal and over-complete representations on a limited dataset of speech and musical test signals. Vincent and Gribonval [5] measured the performance of separation by binary masking using MDCT bases and blind/oracle cosine and wavelet packets. The purpose of the test was to see whether an adaptive transform like cosine packets provides better separability than a fixed one like MDCT. The results showed that only cosine packets offered a slight improvement over MDCT and this might not justify the computationally intensive best basis selection step required for acquiring an orthogonal basis. Also Yilmaz and Rickard [1] present measurements on the performance of binary masks for speech signals, created using knowledge of the STFT magnitudes of the source and interference, suggesting that speech can be considered highly disjoint. Our main contribution is to use a database of real music recordings that span a wide range of popular musical genres and to extend the analysis to a set of popular transforms, including STFT, constant Q transform [6] and pitch synchronous lapped orthogonal transforms [7], with the purpose of measuring the influence of the transform on the disjointness of the representation.

\section{MASKING IN THE TRANSFORM DOMAIN}

Let us consider a linear sum of $K$ sources $\mathbf{s}_{k}: \mathbf{x}=\sum_{k=1}^{K} \mathbf{s}_{k}$, where scaling factors for the sources are absorbed in the source signals for convenience. The linearly transformed mixture can be written as:

$$
\mathcal{T}(\mathbf{x})=\sum_{k=1}^{K} \mathcal{T}\left(\mathbf{s}_{k}\right)
$$

In the present work we exploit knowledge about the sources be- 
fore mixing to calculate "oracle" masks for the sources that are optimal in terms of W-disjointness orthogonality. The ideal masking for a concerned source specifies the regions in the time-frequency representation domain where the signal energy of that source is greater than the interfering energy from all the other sources in that region. That way, we can test the capabilities of a transform without suffering any loss in performance from estimating the masks.

The ideal binary mask for each source of the mixture in the transform domain is calculated as:

$$
M_{k}(\gamma)=\left\{\begin{array}{lc}
1, & \text { for }\left|S_{k}(\gamma)\right|>\left|Y_{k}(\gamma)\right| \\
0, & \text { otherwise }
\end{array}\right.
$$

where $S_{k}(\gamma)=\mathcal{T}\left(\mathbf{s}_{k}\right), \gamma \in \Gamma$ denotes elements from the set of coefficients in the transform domain and $\mathbf{y}_{k}=\sum_{j \neq k} \mathbf{s}_{j}$ is the sum of the sources interfering with the k-th source in the mixture. In the special case where the transform results in a time-frequency representation of the signal, $\gamma \in \Gamma$ can be parametrized by $\gamma=(\omega, t)$ where $\omega$ and $t$ are the frequency and time indexes respectively. The mask is then defined as $M_{k}(\omega, t)$ and indicates the time-frequency points where $\mathbf{s}_{k}$ has more energy than the combined energy of all the interfering sources.

In the ideal case where for different time-frequency regions there is only one source contributing to the mixture, sources can be considered as perfectly disjoint. Then it is be possible to partition the support of the mixture in such a way as to obtain the original sources simply by applying the appropriate binary mask to the mixture. In a less ideal and more realistic setting we can measure the degree of disjointness between the sources. For a measure of disjointness we use the approximate W-disjoint orthogonality (WDO), where $W$ stands for the analysis window function of the transform, as proposed by Yilmaz and Rickard in [1]. WDO with oracle masks provides an upper limit for source separation performance using binary masks (See Appendix A)

\section{TRANSFORMS}

The following transforms were tested and compared in terms of the sparsity and disjointness of their representations.

- Short time Fourier transform (STFT) was employed using Hamming window with $50 \%$ overlap, a common choice of parameters that ensure the invertibility of the transform using inverse Fourier transform and overlap-add synthesis.

- Constant Q transform is a time-scale representation, similar to a wavelet transform but with higher frequency resolution than in conventional wavelet transforms [6]. The frequency bins are logarithmically distributed so that the Q-factors (ratios of the centre frequencies to bandwidths) are the same for all bins.

- Modified discrete cosine transform (MDCT) is a time-frequency transform widely used in coding standards such as MP3. It belongs to a flexible class of transforms called Lapped orthogonal transforms (LOTs) [7]. Here MDCT with type-IV bases is used.

- Pitch-synchronous STFT is realised as a particular instance of LOTs, where the time domain signal is analysed using rectangular windows (with zero overlap) whose lengths are adapted to the pitch (See Appendix B). We also implemented LOTs with Fourier and DCT-IV bases with overlapping pitch-synchronous windows, but the performance of these was slightly worse to that of STFT and MDCT respectively, therefore results are not shown.

\section{EXPERIMENT}

We used a dataset of 18 multitrack songs of varied genre from poprock to heavy metal so as to have a representative and varied set of modern popular music. The songs were sampled at $44.1 \mathrm{kHz}$. The tracks we focused on per song were: Guitar, Bass, Drums, and Vocals. For measuring the disjointness, the measurements were performed on random 2.9-sec segments from the songs, that were energy-normalized. During the selection, we used the following criterion to ensure that none of the sources to be measured were silent in the segment. A random segment $\mathbf{x}_{i}$ was only considered as valid and was used in the measurements if the RMS value of each source signal present in segment $i$ of the mixture was no more than $20 \mathrm{~dB}$ lower than the RMS level of the whole mix.

We collected WDO measurements, according to Eq. (4) in Appendix A, for 30 such segments per song and obtained values for all possible different pairs of instruments in order to show how disjoint they are from one another. Furthermore, we introduce a pitchsynchronous analysis prior to the transform in order to improve the sparsity of the representation of the sources and thus improve their separability. Motivated by the fact that Western music favours harmonic pitch relationships for simultaneously sounding notes, we applied the pitch estimation algorithm on a single track and then measure the disjointness by applying a pitch-synchronous STFT on the mixture with analysis windows adapted to the pitch of one of the sources present in the mixture (see appendix B). The pitch estimator was applied on the Bass track and from the estimated pitches we defined the analysis window lengths of non-overlapping rectangular windows for the STFT of the instrument tracks prior to measuring their disjointness. The reason why we chose to perform the pitchsynchronous analysis on the Bass track is mainly due to the fact that the signal is monophonic (single-pitched) and "dry" (no effects applied) in most of the data.

\section{RESULTS}

Figure 1 present pairwise WDO measurements for all pairs of instruments for the following transforms: STFT, pitch-synchronous STFT, MDCT, and CQT, for different resolutions. The results show significant variance in the data, but this is mainly due to the wide variety of music tracks used for the experiment. The disjointness between two instruments depends highly on the genre, the artist but also on the musical structure of the song. Nevertheless, relative differences in WDO between different transforms and resolutions for an individual song followed rather closely the median across all songs, despite the differences in the absolute song-dependent WDO values. To clarify this, Figure 2 illustrates ratios of WDO values of songs relative to $1024 \mathrm{MDCT}$. Here the variances are much lower.

Another interesting observation is that different pairs of instruments exhibit different levels of disjointness that are partly explained by the frequency range of some instruments (bass in particular) but also by the formant structure of the vocals and note structures of the guitar and bass). Furthermore, measurements (omitted here due to space limitations) showed that when all instruments are present simultaneously, there is considerably more overlap, the disjointness of individual instruments in the mixture reducing to $66.6 \%, 67.7 \%, 70,9 \%$ and $81 \%$ for guitar, bass, drums and vocals, respectively, for STFT and $93 \mathrm{~ms}$ window. This can be contrasted with the $83.4 \%$ WDO obtained for a 4 speaker speech mixtures in [1].

Among pitch-synchronous LOTs, pitch-sync STFT with zero 


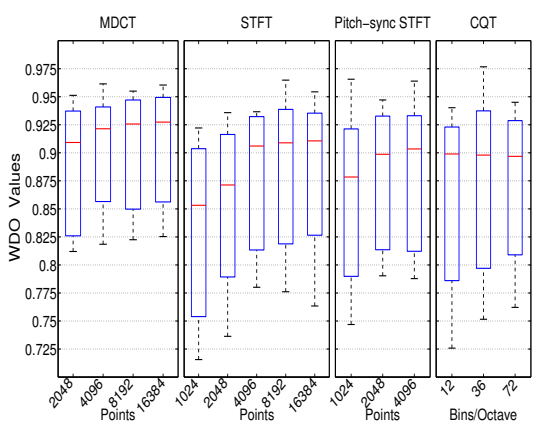

(a) Guitar/Bass

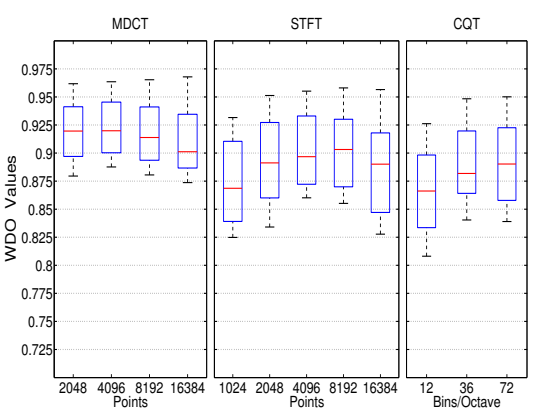

(d) Guitar/Vocals

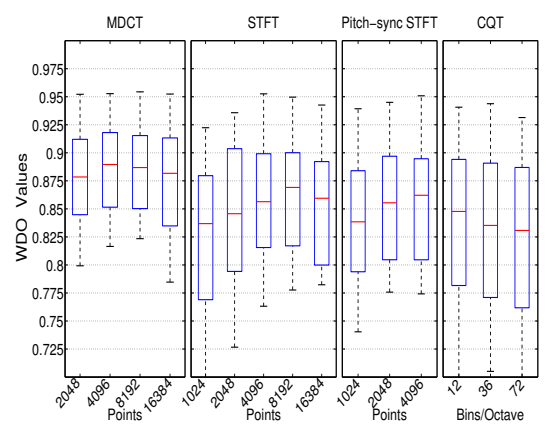

(b) Drums/Bass

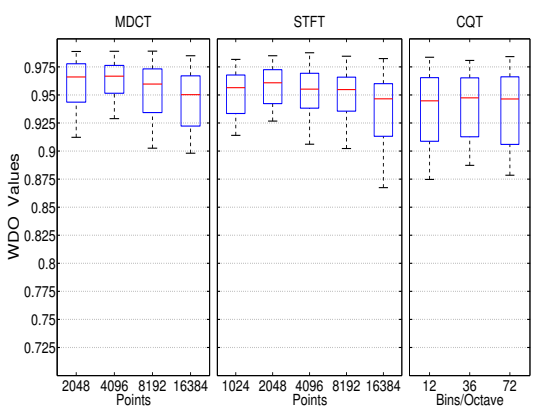

(e) Drums/Vocals

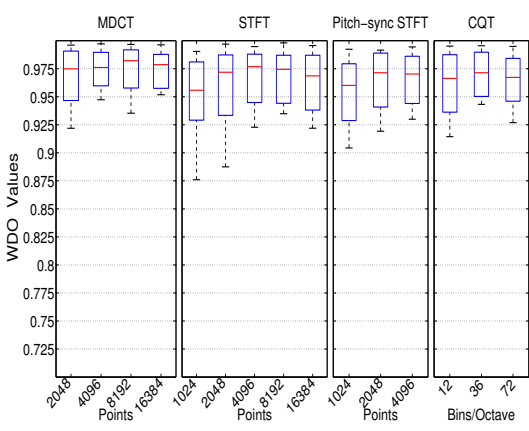

(c) Bass/Vocals

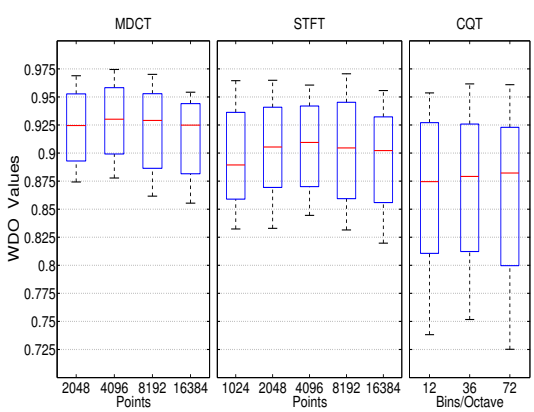

(f) Guitar/Drums

Figure 1: $W D O$ measurements of different pairs of instruments for various transforms

overlap and rectangular window had the best performance imrpovement and therefore results are shown only for that. This could probably be explained due to windowing smearing the spectral peaks in the transform domain, but further experiments are required to better understand the behaviour of pitch-synchronous transforms.

We found no correlation between the disjointess and the sparsity of a representation. Figure 3 shows the sparsity vs. disjointness measurements for different instrument pairs in each of the 18 songs. Sparsity was measured using $\ell_{1} / \ell_{2}$-norm, that is, calculating $\ell_{1}$ norm after normalizing by the $\ell_{2}$ norm for each time frame and then averaging over all framers. The transform used here was MDCT with 4096 points. The sparsity and disjointness values for each mixture were obtained by calculating $\ell_{1} / \ell_{2}$ and WDO separately for the two instrument signals and calculating the average respectively. The figure shows clearly that sparsity does not imply disjointness and vice versa in music.

\section{CONCLUSIONS AND FUTURE WORK}

In this paper we studied the level of disjointness of different instruments in music recordings for various time-frequency representations. The results show that different pairs of instruments exhibit different levels of disjointness, and on the average, MDCT outperformed STFT and CQT. Analysis window length of $93 \mathrm{~ms}$ gave the best results, with a few exceptions where longer analysis windows performed slightly better, mainly when dealing with pairs of pitched instrument tracks and especially in the case of the bass. Pitchsynchronous analysis was introduced to obtain sparser representations and to study the difference this made to the disjointness levels. Although the performance was improved for transforms with short analysis window lengths when dealing with pairs of pitched instruments (such as guitar/bass), this improvement disappears for longer

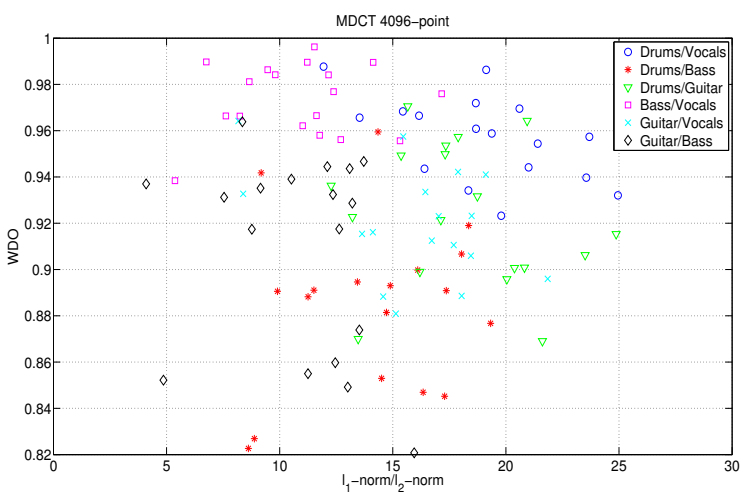

Figure 3: Sparsity vs Disjointness of the different pairs of instruments for the 4096-point MDCT

windows. Further experiments are needed to support this finding.

In future work, we will explore the disjointness of overcomplete representations, including dictionary-learning approaches and unions of bases, each with different time-frequency characteristics [8]. Subsequent experiments could also measure the efficiency of a pitch synchronous analysis in a realistic scenario where the individual sources will not be available.

\section{REFERENCES}

[1] Ö. Yılmaz and S. Rickard, "Blind separation of speech mixtures via time-frequency masking," IEEE Trans. on Signal Processing, vol. 52, no. 7, pp. 1830-1847, Jul. 2004.

[2] A. Ozerov, P. Philippe, F. Bimbot, and R. Gribonval, "Adaptation of bayesian models for single-channel source separation and its application to voice/music separation in popular 


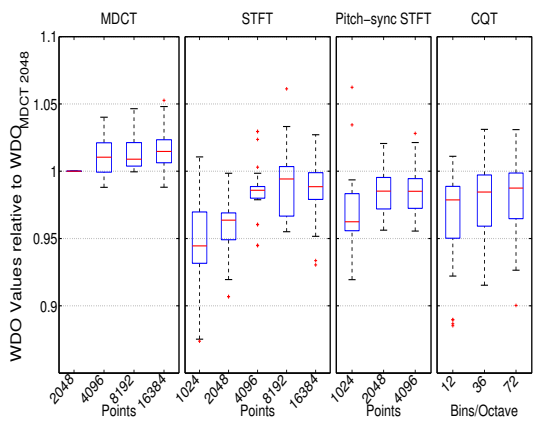

(a) Guitar/Bass

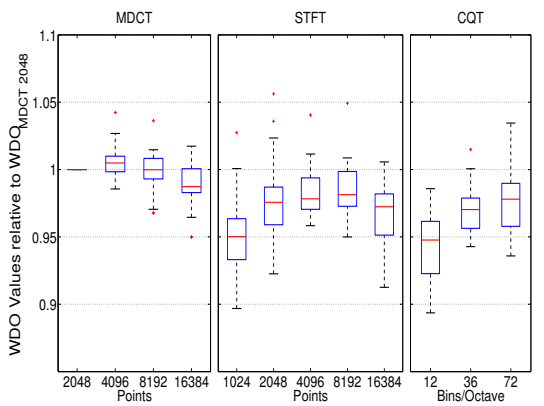

(d) Guitar/Vocals

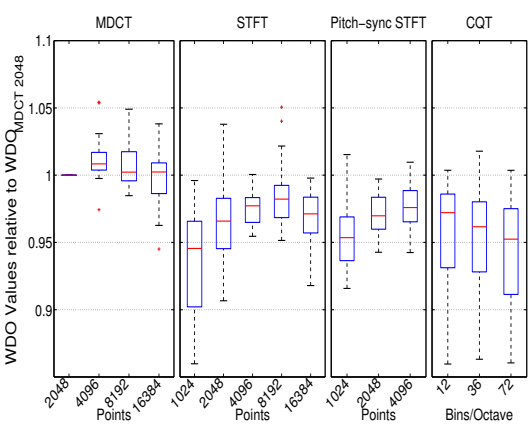

(b) Drums/Bass

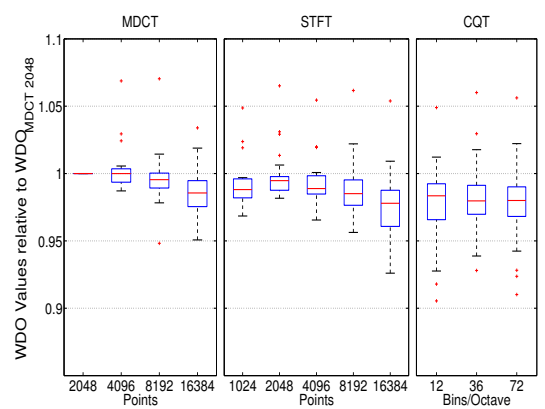

(e) Drums/Vocals

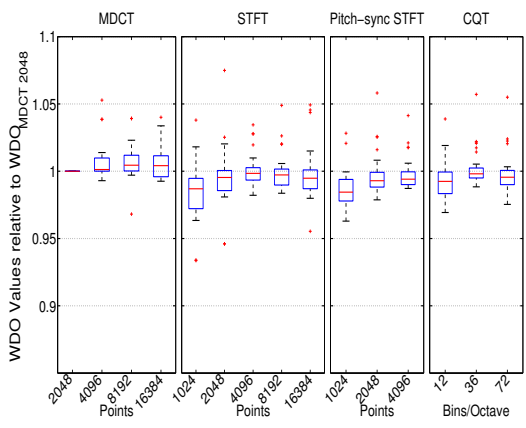

(c) Bass/Vocals

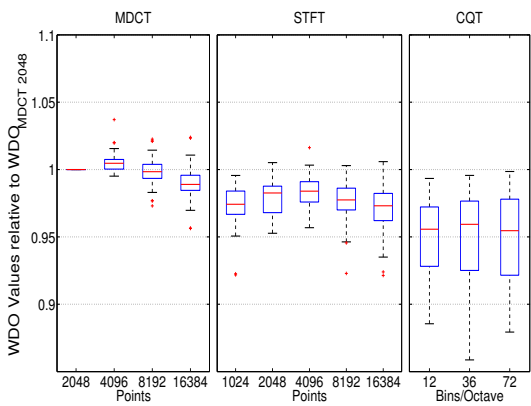

(f) Guitar/Drums

Figure 2: Ratios of $W D O$ measurements relative to $W D O_{\mathrm{MDCT} 1024}$ for different pairs of instruments

songs," IEEE Trans. on Audio, Speech and Language Processing, vol. 15, no. 5, pp. 1564-1578, 2007.

[3] G. Mysore, "A non-negative framework for joint modeling of spectral structure and temporal dynamics in sound mixtures," Ph.D. dissertation, Stanford University, 2010.

[4] V. Y. F. Tan and C. Févotte, "A study of the effect of source sparsity for various transforms on blind audio source separation performance," in Proc. of SPARS05, 2005, pp. 16-19.

[5] E. Vincent and R. Gribonval, "Blind criterion and oracle bound for instantaneous audio source separation using adaptive timefrequency representations," in IEEE WASPAA, 2007, pp. 110 113.

[6] C. Schörkhuber and A. Klapuri, "Constant-Q transform toolbox for music processing," in Sound and Music Computing Conference, Barcelona, 2010.

[7] H. S. Malvar, Signal Processing with Lapped Transforms. Artech Print on Demand, 1992.

[8] M. D. Plumbley, T. Blumensath, L. Daudet, R. Gribonval, and M. Davies, "Sparse representations in audio and music: From coding to source separation," Proceedings of the IEEE, vol. 98, no. 6, pp. 995-1005, Jun. 2010.

[9] A. Klapuri and T. Virtanen, "Representing musical sounds with an interpolating state model," IEEE Trans. on Audio, Speech and Language Processing, vol. 18, no. 3, Mar. 2010.

\section{A. W-DISJOINT ORTHOGONALITY}

Sources are considered pairwise disjoint orthogonal on a transform domain $\mathcal{T}$ if the supports of the transforms of the sources are disjoint. The following method as defined in [1] can measure the level of overlap between the representations of sources using ideal binary masks created based on prior information of the sources.

Given a mask $M$, such that $0 \leq M(\gamma) \leq 1$ for all elements $\gamma$ in the transform space $\Gamma$, the preserved-signal ratio $\left(P S R_{M}\right)$ and the signal-to-interference ratio $\left(S I R_{M}\right)$ performance criteria are defined as:

$$
P S R_{M}=\frac{\left\|M(\gamma) S_{k}(\gamma)\right\|^{2}}{\left\|S_{k}(\gamma)\right\|^{2}}, \operatorname{SIR}_{M}=\frac{\left\|M(\gamma) S_{k}(\gamma)\right\|^{2}}{\left\|M(\gamma) Y_{k}(\gamma)\right\|^{2}}
$$

The approximate $\mathrm{W}$-disjoint orthogonality is then defined as:

$$
W D O_{M}=P S R_{M}-\frac{P S R_{M}}{S I R_{M}}
$$

The maximum possible value, $W D O_{M_{k}}=1$, implies that the mask $M_{k}$ can perfectly separate and recover the k-th source.

\section{B. PITCH SYNCHRONOUS ANALYSIS}

It is well known that Fourier-like transforms of periodic signals are sparser if the length of the analysis window is a multiple of the period length. In order to adapt the analysis window length to the local fundamental period, we run a pitch estimation algorithm prior to the transforms. For improved accuracy, over the pitch estimation we employed the following method, as used in [9]. We first conduct a preliminary pitch analysis where pitch is estimated in $93 \mathrm{~ms}$ time frames with $75 \%$ overlap. Following this, pitch markers that indicate each individual period were generated using dynamic programming to fine-tune the preliminary pitch estimates. The transform window lengths were then chosen so that they would consist of a discrete number of pitch periods as close to a predefined reference window length $(1024,2048$ or 4096) as possible. Furthermore, by exploiting LOTs, we can vary various other parameters such as window edges(tail) lengths and types while ensuring critical sampling. 